\title{
Scalar perturbations in cosmological $f(R)$ models: the cosmic screening approach
}

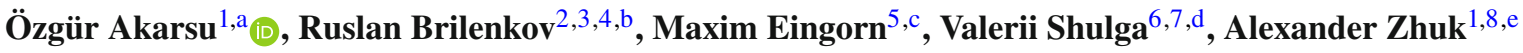 \\ ${ }^{1}$ Department of Physics, Istanbul Technical University, 34469 Maslak, Istanbul, Turkey \\ ${ }^{2}$ Institute for Astro- and Particle Physics, University of Innsbruck, Technikerstrasse 25/8, A-6020 Innsbruck, Austria \\ ${ }^{3}$ Dipartimento di Fisica, Università di Roma 'Tor Vergata', via della Ricerca Scientifica 1, 00133 Rome, Italy \\ ${ }^{4}$ Institut für Astrophysik, Georg-August-Universität Göttingen, Friedrich-Hund-Platz 1, 37077 Göttingen, Germany \\ 5 Department of Mathematics and Physics, North Carolina Central University, Fayetteville st. 1801, Durham, NC 27707, USA \\ ${ }^{6}$ International Center of Future Science of the Jilin University, 2699 Qianjin St., Changchun City 130012, China \\ ${ }^{7}$ Institute of Radio Astronomy of National Academy of Sciences of Ukraine, 4 Mystetstv str., Kharkiv 61002, Ukraine \\ ${ }^{8}$ Astronomical Observatory, Odessa National University, Dvoryanskaya st. 2, Odessa 65082, Ukraine
}

Received: 8 June 2018 / Accepted: 19 July 2018 / Published online: 30 July 2018

(C) The Author(s) 2018

\begin{abstract}
We investigate cosmological perturbations for nonlinear $f(R)$ models within the cosmic screening approach. Matter is considered both in the form of a set of discrete pointlike massive bodies and in the form of a continuous pressureless perfect fluid. We perform full relativistic analysis of the first-order theory of scalar perturbations for arbitrary nonlinear $f(R)$ models and demonstrate that scalar potentials $\Phi(t, \mathbf{r})$ and $\Psi(t, \mathbf{r})$ are determined by a system of only two master equations. Our equations are applicable at all spatial scales as long as the approximation $\delta R / \bar{R} \ll 1$ (which is usually assumed in studies devoted to cosmological perturbations in $f(R)$ models) works.
\end{abstract}

\section{Introduction}

Since the discovery of the late time accelerated expansion of our Universe at the end of the last century, the mystery of dark energy remains one of the most intriguing subjects in modern cosmology. One of the possible solutions, alternative to the introduction of dark energy, is to modify gravity, namely, the general theory of relativity. This idea was known from the eighties of the last century, when $R$ of Einstein-Hilbert action replaced by $R+\alpha R^{2}$ of Starobinsky action was considered to describe the accelerated expansion of the early Universe (so

\footnotetext{
a e-mail: akarsuo@itu.edu.tr

b e-mail: ruslan.brilenkov@gmail.com

c e-mail: maxim.eingorn@gmail.com

de-mail: shulga@rian.kharkov.ua

e e-mail: ai.zhuk2@gmail.com
}

called inflation) [1]. ${ }^{1}$ It was then realized that nonlinear $f(R)$ models can also describe the late acceleration. This resulted in a huge number of articles devoted to the study of nonlinear gravitational models (see, e.g., reviews [3-10]). Because the nature of dark energy is not clear yet, these theories are still of unflagging interest (see, e.g., the most recent papers [1114]).

Obviously, the observable large scale structure of the Universe is the crucial test of any gravitational theory. Comparing the predictions of such a theory with the observational picture, we can conclude how viable it is. This can be done within the framework of perturbation theory. Usually, the Friedmann Universe is considered as a background cosmological model. For such background in the case of the first-order theory of scalar perturbations, the metric perturbations are characterized by two functions $\Phi$ and $\Psi[15,16]$, where the former one is the gravitational potential created by inhomogeneities. For $f(R)$ models, the system of equations for $\Phi$ and $\Psi$ was obtained in [17,18] (see also [4]). The energy density fluctuation $\delta \varepsilon=-\delta T_{0}^{0}$ is the source of the potentials $\Phi$ and $\Psi$ in this system. ${ }^{2}$ However, since the energy-momentum tensor $T^{\alpha \beta}$ depends on metric, the energy density fluctuation is a function of potentials $\Phi$ and $\Psi$. For example, in the linear gravity model the term proportional to $\bar{\varepsilon} \Phi$ contributes to $\delta \varepsilon$ (see also Eq. (2.17) below), where $\bar{\varepsilon}$ is the averaged energy density. In the literature which we are aware of and which is devoted to the study of perturbations in nonlinear mod-

\footnotetext{
$\overline{1}$ One can also see [2] for a possibility of inflation from quadratic and quartic nonlinearities in multidimensional cosmological models.

${ }^{2}$ In the case of linear gravity (i.e. $f(R)=R$ ) with matter in the form of a perfect fluid, the potentials are equal to each other: $\Phi=\Psi$.
} 
els (see, for example, reviews [3-10] and references therein, in particular, the paper [19]), the explicit dependence of the energy-momentum tensor on the perturbations of the metric is not taken into account. This is one of the main differences between our approach and these articles. This drastically changes the form of the gravitational interaction. As it was shown in our papers [20-23], the gravitational potential satisfies the Helmholtz-type equation but not the Poisson equation. Therefore, it has the form of the Yukawa potential. It is important to note that the cosmological background in the form of the Friedmann Universe with the nonzero averaged energy density $\bar{\varepsilon} \neq 0$ plays the crucial role in this effect. In the case of the Minkowski background $\bar{\varepsilon}=0$, and we go back to the Poisson equation which has a solution in the form of Newtonian gravitational potential in full agreement with the textbook [24]. Since the gravitational potential of an individual inhomogeneity is exponentially suppressed at large cosmological scales, we call this effect a cosmic screening $[21,25] .{ }^{3}$ It is worth noting that nonlinearity of gravity in $f(R)$ models also affects the form of the gravitational potential [4]. For example, at the astrophysical scales the Newtonian gravitational potential acquires an additional term in the form of the Yukawa potential [32,33].

In the present paper we study the first-order scalar cosmological perturbations for nonlinear $f(R)$ models within the cosmic screening approach. We demonstrate that in this case a rather complicated initial system of six differential equations is reduced to two equations for the potentials $\Phi$ and $\Psi$. These equations establish the basis for numerical simulation of the large-scale structure of the Universe in nonlinear gravity models.

The paper is structured as follows. In Sect. 2, we describe the model and present the basic equations at the background and perturbation levels. In Sect. 3, we obtain two master equations for the potentials $\Phi$ and $\Psi$. The main results are briefly summarized in the concluding Sect. 4.

\section{The model and basic equations}

In this section we present the basic equations that we will use hereinafter. For background and perturbed equations we follow mainly the review [4] using the sign convention accepted in this paper. In $f(R)$ gravity, the action reads

\footnotetext{
${ }^{3}$ In our previous mechanical approach [26-29], we also took into account the dependence of the perturbed energy density on the gravitational potential. However, since we dropped the additional term $\bar{\varepsilon} \Phi$ in the perturbed Einstein equation for $\Phi$, the exponential suppression of gravitational interaction was not observed in this approach. The theory of scalar perturbations for nonlinear $f(R)$ models within the mechanical approach was studied in the paper [30]. For the linear $f(R)=R$ model, the second-order cosmological perturbations within the cosmic screening approach were studied in [31].
}

$S=\frac{1}{2 \kappa^{2}} \int d^{4} x \sqrt{-g} f(R)+S_{m}$

Here $f(R)$ is an arbitrary function of the scalar curvature $R$, $S_{m}$ is the action for matter, $\kappa^{2} \equiv 8 \pi G_{N}$, where $G_{N}$ stands for Newtonian gravitational constant, and we use units such that the speed of light is equal to $1: c \equiv 1$. The equation of motion corresponding to this action is

$$
\begin{aligned}
& F(R) R_{\mu \nu}-\frac{1}{2} f(R) g_{\mu \nu}-\nabla_{\mu} \nabla_{\nu} F(R)+g_{\mu \nu} \square F(R) \\
& =\kappa^{2} T_{\mu \nu} \quad \mu, \nu=0,1,2,3 .
\end{aligned}
$$

The trace of this equation gives

$3 \square F(R)+F(R) R-2 f(R)=\kappa^{2} T$,

where $T=g^{\mu \nu} T_{\mu \nu}$ and $F(R) \equiv f^{\prime}(R)$. Hereinafter, the prime denotes the derivative with respect to the scalar curvature $R$, i.e. $f^{\prime}(R) \equiv d f / d R$. Since $f(R)$ has dimension of $R$, the function $F(R)$ is dimensionless. Besides, $\square F=(1 / \sqrt{-g}) \partial_{\mu}\left(\sqrt{-g} g^{\mu \nu} \partial_{\nu} F\right)$.

In the case of the spatially flat background spacetime with Friedmann-Robertson-Walker (FRW) metric

$d s^{2}=g_{\mu \nu} d x^{\mu} d x^{\nu}=-d t^{2}+a^{2}(t)\left(d x^{2}+d y^{2}+d z^{2}\right)$

and matter in the form of a perfect fluid with the energymomentum tensor components $\bar{T}_{v}^{\mu}=\operatorname{diag}(-\bar{\varepsilon}, \bar{P}, \bar{P}, \bar{P})$, Eq. (2.2) results in the following system of background equations:

$3 F H^{2}=(F R-f) / 2-3 H \dot{F}+\kappa^{2} \bar{\varepsilon}$

and

$-2 F \dot{H}=\ddot{F}-H \dot{F}+\kappa^{2}(\bar{\varepsilon}+\bar{P})$.

Here the bar denotes homogeneous background quantities, the Hubble parameter $H \equiv \dot{a} / a$ (the dot everywhere denotes the derivative with respect to the synchronous time $t$ ) and the scalar curvature

$R=6\left(2 H^{2}+\dot{H}\right)$.

The perfect fluid satisfies the continuity equation

$\dot{\bar{\varepsilon}}+3 H(\bar{\varepsilon}+\bar{P})=0$,

which for nonrelativistic matter with $P=0$ has the solution

$\bar{\varepsilon}=\bar{\rho} / a^{3}$, 
where $\bar{\rho}=$ const is the averaged mass density in the comoving coordinates. In what follows we will consider matter either in the form of a set of discrete point-like massive bodies or in the form of a continuous pressureless perfect fluid. For both of these cases the pressure and its fluctuation are equal to zero: $P=\delta P=0$.

Let us turn now to the perturbed equations. Inhomogeneities or fluctuations of matter result in perturbations of the FRW metric (2.4). In our paper we restrict ourselves to the scalar perturbations. In the conformal Newtonian (longitudinal) gauge, the perturbed metric reads $[15,16]$

$$
d s^{2}=-(1+2 \Phi) d t^{2}+a^{2}(1-2 \Psi)\left(d x^{2}+d y^{2}+d z^{2}\right),
$$

where the introduced scalar perturbations $\Phi, \Psi \ll 1$ depend on all spacetime coordinates. These perturbations satisfy the following system of linearized Eqs. [4]:

$$
\begin{aligned}
& -\frac{\Delta \Psi}{a^{2}}+3 H(H \Phi+\dot{\Psi}) \\
= & -\frac{1}{2 F}\left[\left(3 H^{2}+3 \dot{H}+\frac{\triangle}{a^{2}}\right) \delta F\right. \\
& \left.-3 H \dot{F}+3 H \dot{F} \Phi+3 \dot{F}(H \Phi+\dot{\Psi})+\kappa^{2} \delta \varepsilon\right], \\
& H \Phi+\dot{\Psi}=\frac{1}{2 F}\left(\dot{\delta}-H \delta F-\dot{F} \Phi-\frac{\kappa^{2}}{a^{2}} \Xi\right) \\
& -F(\Phi-\Psi)=\delta F \\
& 3(\dot{H} \Phi+H \dot{\Phi}+\ddot{\Psi})+6 H(H \Phi+\dot{\Psi}) \\
& +3 \dot{H} \Phi+\frac{\triangle \Phi}{a^{2}} \\
= & \frac{1}{2 F}\left[3 \ddot{F}+3 H \dot{F}-6 H^{2} \delta F-\frac{\triangle \delta F}{a^{2}}-3 \dot{F} \dot{\Phi}\right. \\
& \left.-3 \dot{F}(H \Phi+\dot{\Psi})-(3 H \dot{F}+6 \ddot{F}) \Phi+\kappa^{2} \delta \varepsilon\right] \\
\delta F= & F^{\prime} \delta R, \delta R=-2[3(\dot{H} \Phi+H \dot{\Phi}+\ddot{\Psi}) \\
& \left.+12 H(H \Phi+\dot{\Psi})+\frac{\Delta \Phi}{a^{2}}+3 \dot{H} \Phi-2 \frac{\Delta \Psi}{a^{2}}\right] \\
+ & \dot{F}(3 H \Phi+3 \dot{\Psi}+\dot{\Phi}) \\
+ & 3 H \dot{F} \Phi-\frac{1}{3} F \delta R \\
a^{2} & -\frac{1}{3} R \delta F=\frac{1}{3} \kappa^{2} \delta \varepsilon+2 \ddot{F} \Phi
\end{aligned}
$$

In these equations the function $F$, its derivative $F^{\prime}$ and the scalar curvature $R$ are unperturbed background quantities, i.e. here $F(R) \equiv F(\bar{R})$. This system of equations is usually used in papers devoted to cosmological perturbations in $f(R)$ models. It is important to note here that the relation $\delta F=F(R)-F(\bar{R})=F^{\prime}(\bar{R}) \delta R$ is valid only in approximation $\delta R / \bar{R} \ll 1$. However, this approximation is violated at the late stage of the evolution of the Universe near inhomogeneities, i.e. in the vicinity of galaxies. Therefore the system of Eqs. (2.11)-(2.16) and, consequently, the solutions of this system are only applicable at sufficiently large scales in the late Universe or at all scales in the early Universe.

The energy density fluctuation $\delta \varepsilon$ reads

$\delta \varepsilon=\frac{\delta \rho(t, \mathbf{r})}{a^{3}}+\frac{3 \bar{\rho}}{a^{3}} \Psi$,

where $\mathbf{r}$ is a comoving radius-vector. For the linear $f(R)=R$ model the potentials $\Psi$ and $\Phi$ are equal to each other: $\Psi=\Phi$. Then, Eq. (2.17) reproduces the result of papers [20-22]. In the case of inhomogeneities in the form of discrete point-like masses $m_{p}$, this equation can be directly obtained from the energy-momentum tensor [24]

$T^{i k}=\sum_{p} \frac{m_{p}}{\sqrt{-g}} \frac{d x_{p}^{i}}{d t} \frac{d x_{p}^{k}}{d t} \frac{1}{d \tau_{p} / d t} \delta\left(\mathbf{r}-\mathbf{r}_{p}\right)$,

where $d \tau^{2}=-d s^{2}$ and the metric is given by (2.10). The fluctuation of the mass density is

$\delta \rho=\rho-\bar{\rho}=\sum_{p} m_{p} \delta\left(\mathbf{r}-\mathbf{r}_{p}\right)-\bar{\rho}$.

In the case of a continuous pressureless perfect fluid with the mass density $\rho(t, \mathbf{r})$, Eq. (2.18) is generalized as follows:

$T^{i k}=\frac{\rho}{\sqrt{-g}} \frac{d x^{i}}{d \tau} \frac{d x^{k}}{d \tau} \frac{d \tau}{d t}=\varepsilon u^{i} u^{k}$,

where

$\varepsilon \equiv \frac{\rho}{\sqrt{-g}} \frac{d \tau}{d t}, \quad u^{i} \equiv \frac{d x^{i}}{d \tau}$

It can be easily seen that for the energy density fluctuation we reproduce Eq. (2.17).

The quantity $\Xi$ describes the effective peculiar velocity potential $[20,21]$. For example, in the case of discrete sources we have

$a \nabla\left(\sum_{p} \rho_{p} \mathbf{v}_{p}\right)=\triangle \Xi$, 
where $\rho_{p} \equiv m_{p} \delta\left(\mathbf{r}-\mathbf{r}_{p}\right)$ and $v_{p}^{\alpha} \equiv d x_{p}^{\alpha} / d t, \alpha=1,2,3$.

This equation can be solved exactly [20]:

$\Xi=\frac{a}{4 \pi} \sum_{p} m_{p} \frac{\left(\mathbf{r}-\mathbf{r}_{p}\right) \mathbf{v}_{p}}{\left|\mathbf{r}-\mathbf{r}_{p}\right|^{3}}$

For the continuous perfect fluid with the energy density (2.21) the function $\Xi$ satisfies the equation

$a \nabla(\rho \mathbf{v})=\triangle \Xi$

\section{Master equations for the potentials $\Phi$ and $\Psi$}

We have six Eqs. (2.11)-(2.16) for two functions $\Phi(t, \mathbf{r})$ and $\Psi(t, \mathbf{r})$. The main goal now is to maximally simplify this system by reducing the number of equations. As will be seen in what follows, we obtain a system of only two independent differential equations, which we call the master equations for $\Phi$ and $\Psi$.

First, substituting (2.13) into Eqs. (2.11), (2.12), (2.14), (2.15) and (2.16), we obtain respectively:

$$
\begin{aligned}
& -\frac{\Delta \Psi}{a^{2}}+3 H(H \Phi+\dot{\Psi}) \\
= & -\frac{1}{2 F}\left[-\left(3 H^{2}+3 \dot{H}+\frac{\triangle}{a^{2}}\right) F(\Phi-\Psi)\right. \\
& +3 H(\dot{F}(\Phi-\Psi)+F(\dot{\Phi}-\dot{\Psi}))+3 H \dot{F} \Phi \\
& \left.+3 \dot{F}(H \Phi+\dot{\Psi})+\kappa^{2} \delta \varepsilon\right], \\
H \Phi+\dot{\Psi}= & \frac{1}{2 F}[-(\dot{F}(\Phi-\Psi)+F(\dot{\Phi}-\dot{\Psi})) \\
& \left.+H F(\Phi-\Psi)-\dot{F} \Phi-\frac{\kappa^{2}}{a^{2}} \Xi\right], \\
& 3(\dot{H} \Phi+H \dot{\Phi}+\ddot{\Psi})+6 H(H \Phi+\dot{\Psi})+3 \dot{H} \Phi+\frac{\triangle \Phi}{a^{2}} \\
= & \frac{1}{2 F}[-3(\ddot{F}(\Phi-\Psi)+2 \dot{F}(\dot{\Phi}-\dot{\Psi})+F(\ddot{\Phi}-\ddot{\Psi})) \\
& -3 H(\dot{F}(\Phi-\Psi)+F(\dot{\Phi}-\dot{\Psi}))+6 H^{2} F(\Phi-\Psi) \\
& +\frac{F}{a^{2}}(\triangle \Phi-\Delta \Psi)-3 \dot{F} \dot{\Phi}-3 \dot{F}(H \Phi+\dot{\Psi}) \\
& \left.-(3 H \dot{F}+6 \ddot{F}) \Phi+\kappa^{2} \delta \varepsilon\right], \\
& -(\ddot{F}(\Phi-\Psi)+2 \dot{F}(\dot{\Phi}-\dot{\Psi})+F(\ddot{\Phi}-\ddot{\Psi})) \\
& -3 H(\dot{F}(\Phi-\Psi)+F(\dot{\Phi}-\dot{\Psi}))+\frac{F}{a^{2}}(\Delta \Phi-\Delta \Psi) \\
& +\frac{1}{3} R F(\Phi-\Psi)=\frac{1}{3} \kappa^{2} \delta \varepsilon+\dot{F}(3 H \Phi+3 \dot{\Psi}+\dot{\Phi}) \\
& +2 \ddot{F} \Phi+3 H \dot{F} \Phi+\frac{2}{3} F[3(\dot{H} \Phi+H \dot{\Phi}+\ddot{\Psi}) \\
& \left.+12 H(H \Phi+\dot{\Psi})+\frac{\Delta \Phi}{a^{2}}+3 \dot{H} \Phi-2 \frac{\Delta \Psi}{a^{2}}\right], \\
& -F(\Phi-4)
\end{aligned}
$$

$$
\left.+12 H(H \Phi+\dot{\Psi})+\frac{\triangle \Phi}{a^{2}}+3 \dot{H} \Phi-2 \frac{\triangle \Psi}{a^{2}}\right] .
$$

Next, from Eqs. (3.4) and (3.5) we find:

$$
\begin{aligned}
\ddot{\Psi}= & \frac{F}{6 F^{\prime}}(\Phi-\Psi)-4 H(H \Phi+\dot{\Psi})-\frac{1}{3} \frac{\triangle \Phi}{a^{2}}-2 \dot{H} \Phi \\
& +\frac{2}{3} \frac{\triangle \Psi}{a^{2}}-H \dot{\Phi}
\end{aligned}
$$

and

$$
\begin{aligned}
\ddot{\Phi}= & \dot{\Phi}\left[-3 \frac{\dot{F}}{F}-4 H\right]-\dot{\Psi}\left[\frac{\dot{F}}{F}+H\right]+\Phi\left[-3 \frac{\ddot{F}}{F}-2 \dot{H}\right. \\
& \left.-9 H \frac{\dot{F}}{F}+\frac{1}{3} R-4 H^{2}-\frac{F}{6 F^{\prime}}\right]+\frac{1}{3 a^{2}}(2 \triangle \Phi-\triangle \Psi) \\
& -\Psi\left[-\frac{\ddot{F}}{F}-3 H \frac{\dot{F}}{F}+\frac{1}{3} R-\frac{F}{6 F^{\prime}}\right]-\frac{1}{3 F} \kappa^{2} \delta \varepsilon .
\end{aligned}
$$

Substituting these expressions for $\ddot{\Phi}$ and $\ddot{\Psi}$ into Eq. (3.3), after rather tedious but not complicated calculations we get

$$
\begin{aligned}
& 6 H F \dot{\Phi}+\dot{\Psi}[6 \dot{F}+6 H F] \\
+ & \Phi\left[18 H \dot{F}-F R+18 F H^{2}\right] \\
+ & \Psi\left[-6 H \dot{F}+F R-6 H^{2} F\right] \\
- & 2 \frac{F}{a^{2}}(\triangle \Phi+\triangle \Psi)+2 \kappa^{2} \delta \varepsilon=0 .
\end{aligned}
$$

Equation (3.2) can be written in the form

$$
\begin{aligned}
F \dot{\Phi}= & -F \dot{\Psi}-\Phi[2 \dot{F}+F H] \\
& -\Psi[-\dot{F}+F H]-\frac{\kappa^{2}}{a^{2}} \Xi .
\end{aligned}
$$

After substituting this equation into (3.8) we obtain

$$
\begin{aligned}
6 \dot{\Psi}= & -\frac{1}{\dot{F}} \Phi\left[6 H \dot{F}-F R+12 F H^{2}\right] \\
& -\frac{1}{\dot{F}} \Psi\left[F R-12 H^{2} F\right] \\
& +\frac{2}{\dot{F}} \frac{F}{a^{2}}(\triangle \Phi+\Delta \Psi)-\frac{2}{\dot{F}} \kappa^{2} \delta \varepsilon+\frac{6}{\dot{F}} H \frac{\kappa^{2}}{a^{2}} \Xi .
\end{aligned}
$$

In addition, we substitute (3.10) into (3.8) and after some algebra obtain

$$
\begin{aligned}
6 \dot{\Phi}= & -\Phi\left[12 \frac{\dot{F}}{F}+\frac{F R}{\dot{F}}-12 \frac{H^{2} F}{\dot{F}}\right] \\
& +\Psi\left[6 \frac{\dot{F}}{F}-6 H+\frac{F R}{\dot{F}}-12 \frac{H^{2} F}{\dot{F}}\right] \\
& -\frac{2}{\dot{F}} \frac{F}{a^{2}}(\triangle \Phi+\Delta \Psi)-\left[\frac{6}{F}+\frac{6 H}{\dot{F}}\right] \frac{\kappa^{2}}{a^{2}} \Xi+\frac{2}{\dot{F}} \kappa^{2} \delta \varepsilon .
\end{aligned}
$$

We have Eq. (3.1) which we have not used yet. Substituting Eqs. (3.10) and (3.11) into this equation, we arrive, after 
rather tedious calculations, at a short and simple equation:

$$
\left[6 F H^{2}+3 \dot{H} F-\frac{1}{2} F R\right](\Phi-\Psi)=0,
$$

and this is an identity due to Eq. (2.7). This confirms the consistency of our Eqs. (3.10) and (3.11). Additionally, Eqs. (3.6) and (3.7) are satisfied for $\dot{\Psi}$ and $\dot{\Phi}$ given by (3.10) and (3.11). This can be proved after rather long algebra with the help of Eq. (2.17) for $\delta \varepsilon$, the continuity equations $\dot{\rho}=-\nabla\left(\sum_{p} \rho_{p} \mathbf{v}_{p}\right)$ and $\dot{\rho}=-\nabla(\rho \mathbf{v})$ (for the discrete and continuous inhomogeneities, respectively), the equation of motion $\dot{\Xi}=-H \Xi-\bar{\rho} \Phi / a$ (for both types of inhomogeneities), Eqs. (2.22) or (2.24) and the background Eq. (2.6) (where $\bar{P}=0$ ) and (2.7). Therefore, Eqs. (3.10) and (3.11) are the only independent differential equations for the potentials $\Psi$ and $\Phi$. These master equations can be rewritten in the final form:

$$
\begin{gathered}
\dot{\Psi}-\frac{1}{3} \frac{F}{\dot{F} a^{2}}(\Delta \Phi+\Delta \Psi)+\Phi\left[H-\frac{F \dot{H}}{\dot{F}}\right] \\
+\Psi\left[\frac{F \dot{H}}{\dot{F}}+\frac{\kappa^{2} \bar{\rho}}{\dot{F} a^{3}}\right]=-\frac{1}{3} \frac{\kappa^{2} \delta \rho}{\dot{F} a^{3}}+\frac{H}{\dot{F}} \frac{\kappa^{2}}{a^{2}} \Xi
\end{gathered}
$$

and

$$
\begin{gathered}
\dot{\Phi}+\frac{1}{3} \frac{F}{\dot{F} a^{2}}(\Delta \Phi+\Delta \Psi)-\Psi\left[\frac{\dot{F}}{F}-H+\frac{F \dot{H}}{\dot{F}}+\frac{\kappa^{2} \bar{\rho}}{\dot{F} a^{3}}\right] \\
+\Phi\left[2 \frac{\dot{F}}{F}+\frac{F \dot{H}}{\dot{F}}\right]=\frac{1}{3} \frac{\kappa^{2} \delta \rho}{\dot{F} a^{3}}-\left[\frac{1}{F}+\frac{H}{\dot{F}}\right] \frac{\kappa^{2}}{a^{2}} \Xi .
\end{gathered}
$$

\section{Conclusion}

In this paper, we have considered the theory of scalar perturbations for nonlinear $f(R)$ models. The initial rather complicated system of six Eqs. (2.11)-(2.16) obtained previously in a number of papers (see, e.g., [4]) has been reduced to two independent differential master Eq. (3.13) and (3.14) for two scalar potentials $\Phi$ and $\Psi$. It can be easily verified that in the linear model case $f(R)=R$, where $\Phi=\Psi$, both of these equations are reduced to the following one:

$\triangle \Phi-\frac{3 \kappa^{2} \bar{\rho}}{2 a} \Phi=\frac{\kappa^{2}}{2 a} \delta \rho-\frac{3 \kappa^{2}}{2} H \Xi$,

where the time derivative disappears. This equation exactly coincides with the one in papers [20-22]. It is the Helmholtz equation (not the Poisson equation), resulting in the Yukawatype screening of the gravitational potential produced by matter inhomogeneities [20-23]. This effect is called cosmic screening. The nonzero cosmological background (i.e., $\bar{\rho} \neq 0$ ) is responsible for this effect. In the present paper we have applied the similar approach. Unfortunately, in the case of nonlinear $f(R)$ models we cannot exclude the time derivatives from Eqs. (3.13) and (3.14) to get the pure Helmholtztype equations. However, we have performed the full relativistic analysis of the first-order theory of scalar perturbations for arbitrary nonlinear $f(R)$ models. In the literature, among such models, special attention is drawn to the models that pass to the $\Lambda \mathrm{CDM}$ model. This can occur both at the early and late stages of the Universe evolution. In the early Universe such transition is necessary to reproduce the conventional matter era $[12,34]$. On the other hand, in the late Universe the transition to the de Sitter stage takes place in a number of viable models which possess the stable de Sitter points [4, 11,30,35-39]. Recently, the stable and unstable de Sitter stages for general $f(R)$ models were derived in [40]. It is worth noting that nonlinear gravitational models which can unify the early and late stages of acceleration through the stage of dominance of matter were considered in [41,42].

As we have already mentioned above (see the text after Eq. (2.16)), our equations are applicable for $f(R)$ models at all spatial scales as long as the approximation $\delta R / \bar{R} \ll 1$ works. ${ }^{4}$ From this point of view, a particular interest is taken in a subclass of $f(R)$ models in which this approximation still works at the stage of transition from the $f(R)$ model to the $\Lambda$ CDM model. The theory of scalar perturbations for the $\Lambda \mathrm{CDM}$ model within the cosmic screening approach was investigated in detail in our papers [20-22]. Therefore, for such a subclass of $f(R)$ models, solutions of the equations for the scalar perturbations obtained in the present paper for the $f(R)$ models and in the papers [20-22] for the $\Lambda \mathrm{CDM}$ model can be matched to each other in the stage of transition. Hence, we can continuously describe the large scale structure formation both on $f(R)$ and $\Lambda$ CDM stages.

Acknowledgements ÖA acknowledges support by the Distinguished Young Scientist Award BAGEP of the Science Academy. AZ acknowledges financial support from The Scientific and Technological Research Council of Turkey (TUBITAK) in the scheme of Fellowships for Visiting Scientists and Scientists on Sabbatical Leave (BIDEB 2221). AZ also acknowledges the hospitality of Istanbul Technical University (ITU) where parts of this work were carried out. The work of RB was partially supported by the EMJMD Student Scholarship from Erasmus +: Erasmus Mundus Joint Master Degree programme AstroMundus in Astrophysics.

Open Access This article is distributed under the terms of the Creative Commons Attribution 4.0 International License (http://creativecomm ons.org/licenses/by/4.0/), which permits unrestricted use, distribution, and reproduction in any medium, provided you give appropriate credit to the original author(s) and the source, provide a link to the Creative Commons license, and indicate if changes were made.

Funded by SCOAP . $^{3}$

\footnotetext{
${ }^{4}$ Our investigation was based on Eqs. (2.11)-(2.16) taken from the review [4]. The form of these equations (see, in particular, (2.16)) clearly demonstrates that they are valid under this approximation. Most of the research in the literature devoted to the scalar perturbations in $f(R)$ models is based on this system of equations.
} 


\section{References}

1. A.A. Starobinsky, A new type of isotropic cosmological models without singularity. Phys. Lett. B 91, 99 (1980)

2. T. Saidov, A. Zhuk, Bouncing inflation in nonlinear $R^{2}+R^{4}$ gravitational model. Phys. Rev. D 81, 124002 (2010). arXiv:1002.4138 [hep-th]

3. T.P. Sotiriou, V. Faraoni, $f(R)$ theories of gravity. Rev. Mod. Phys. 82, 451 (2010). arXiv:0805.1726 [gr-qc]

4. A. De Felice, S. Tsujikawa, $f(R)$ theories. Living Rev. Relativ. 13, 3 (2010). arXiv: 1002.4928 [gr-qc]

5. S. Nojiri, S.D. Odintsov, Unified cosmic history in modified gravity: from $\mathrm{F}(\mathrm{R})$ theory to Lorentz non-invariant models. Phys. Rep. 505, 59 (2011). arXiv: 1011.0544 [gr-qc]

6. H. Motohashi, A.A. Starobinsky, J. Yokoyama, $f(R)$ gravity and its cosmological implications. Int. J. Mod. Phys. D 20, 1347 (2011). arXiv:1101.0716 [astro-ph.CO]

7. H. Motohashi, A.A. Starobinsky, J. Yokoyama, $f(R)$ cosmology and massive neutrinos. Int. J. Mod. Phys. Conf. Ser. 10, 35 (2012)

8. K. Koyama, Cosmological tests of modified gravity. Rep. Prog. Phys. 79, 046902 (2016). arXiv:1504.04623 [astro-ph.CO]

9. S. Nojiri, S.D. Odintsov, V.K. Oikonomou, Modified gravity theories on a nutshell:inflation, bounce and late-time evolution. Phys. Rep. 692, 1 (2017). arXiv:1705.11098 [gr-qc]

10. C. Burrage, J. Sakstein, Tests of chameleon gravity. Living Rev. Rel. 21, 1 (2018). arXiv:1709.09071 [astro-ph.CO]

11. S. Capozziello, K.F. Dialektopoulos, O. Luongo, Maximum turnaround radius in $f(R)$ gravity, arXiv: 1805.01233 [gr-qc]

12. A. Peel, V. Pettorino, C. Giocoli, J.L. Starck, M. Baldi, Breaking degeneracies in modified gravity with higher (than 2nd) order weak-lensing statistics, arXiv: 1805.05146 [astro-ph.CO]

13. R.C.C. Lopes, R. Voivodic, L.R. Abramo, L. Sodré, Turnaround radius in $f(R)$ model, arXiv:1805.09918 [astro-ph.CO]

14. M. Roncarelli, M. Baldi, F. Villaescusa-Navarro, The kinematic Sunyaev-Zel'dovich effect of the large-scale structure (II): the effect of modified gravity, arXiv:1805.11607 [astro-ph.CO]

15. V.F. Mukhanov, H.A. Feldman, R.H. Brandenberger, Theory of cosmological perturbations. Part 1. Classical perturbations. Part 2. Quantum theory of perturbations. Part 3. Extensions. Phys. Rep. 215, 203 (1992)

16. D.S. Gorbunov, V.A. Rubakov, Introduction to the Theory of the Early Universe: Cosmological Perturbations and Inflationary Theory (World Scientific, Singapore, 2011)

17. J. Hwang, H. Noh, Gauge ready formulation of the cosmological kinetic theory in generalized gravity theories. Phys. Rev. D 65, 023512 (2002). arXiv:astro-ph/0102005

18. J. Hwang, H. Noh, Classical evolution and quantum generation in generalized gravity theories including string corrections and tachyon: Unified analyses. Phys. Rev. D 71, 063536 (2005). arXiv:gr-qc/0412126

19. A. de la Cruz-Dombriz, A. Dobado, A.L. Maroto, On the evolution of density perturbations in $f(R)$ theories of gravity. Phys. Rev. D 77, 123515 (2008). arXiv:0802.2999 [astro-ph]

20. M. Eingorn, First-order cosmological perturbations engendered by point-like masses. Astrophys. J. 825, 84 (2016). arXiv:1509.03835 [gr-qc]

21. M. Eingorn, C. Kiefer, A. Zhuk, Scalar and vector perturbations in a universe with discrete and continuous matter sources. JCAP 09, 032 (2016). arXiv:1607.03394 [gr-qc]

22. M. Eingorn, R. Brilenkov, Perfect fluids with $\omega=$ const as sources of scalar cosmological perturbations. Phys. Dark Univ. 17, 63 (2017). arXiv:1509.08181 [gr-qc]
23. M. Eingorn, Cosmological law of universal gravitation. Int. J. Mod. Phys. D 26, 1750121 (2017). arXiv:1709.02264 [gr-qc]

24. L.D. Landau, E.M. Lifshitz, The Classical Theory of Fields, Fourth Edition: Volume 2 (Course of Theoretical Physics Series) (Oxford Pergamon Press, Oxford, 2000)

25. M. Eingorn, C. Kiefer, A. Zhuk, Cosmic screening of the gravitational interaction. Int. J. Mod. Phys. D 26, 1743012 (2017). arXiv:1711.01759 [gr-qc]

26. M. Eingorn, A. Zhuk, Hubble flows and gravitational potentials in observable Universe. JCAP 09, 026 (2012). arXiv:1205.2384 [astro-ph.CO]

27. M. Eingorn, A. Zhuk, Remarks on mechanical approach to observable Universe. JCAP 05, 024 (2014). arXiv:1309.4924 [astroph.CO]

28. M. Eingorn, A. Kudinova, A. Zhuk, Dynamics of astrophysical objects against the cosmological background. JCAP 1304, 010 (2013). arXiv:1211.4045 [astro-ph.CO]

29. A. Zhuk, Perfect fluids coupled to inhomogeneities in the late Universe. Grav. Cosmol. 22, 159 (2016). arXiv:1601.01939 [gr-qc]

30. M. Eingorn, J. Novák, A. Zhuk, $f(R)$ gravity: scalar perturbations in the late Universe. Eur. Phys. J. C 74, 3005 (2014). arXiv:1401.5410 [astro-ph.CO]

31. R. Brilenkov, M. Eingorn, Second-order cosmological perturbations engendered by point-like masses. Astrophys. J. 845, 153 (2017). arXiv:1703.10282 [gr-qc]

32. M. Eingorn, A. Zhuk, Weak-field limit of $f(R)$-gravity in three and more spatial dimensions. Phys. Rev. D 84, 024023 (2011). arXiv:1104.1456 [gr-qc]

33. M. Eingorn, A. Zhuk, Asymptotic latent solitons, black strings and black branes in $f(R)$-gravity. Phys. Rev. D 85, 064030 (2012). arXiv: 1112.1539 [gr-qc]

34. R.C. Nunes, S. Pan, E.N. Saridakis, E.M.C. Abreu, New observational constraints on $f(R)$ gravity from cosmic chronometers. JCAP 01, 005 (2017). arXiv: 1610.07518 [astro-ph.CO]

35. G. Cognola, E. Elizalde, S. Nojiri, S.D. Odintsov, S. Zerbini, Oneloop $f(R)$ gravity in de Sitter universe. JCAP 02, 010 (2005). arXiv:hep-th/0501096

36. G. Cognola, E. Elizalde, S.D. Odintsov, P. Tretyakov, S. Zerbini, Initial and final de Sitter universes from modified $f(R)$ gravity. Phys. Rev. D 79, 044001 (2009). arXiv:0810.4989 [gr-qc]

37. K. Bamba, C.Q. Geng, C.C. Lee, Generic feature of future crossing of phantom divide in viable $f(R)$ gravity models. JCAP 11, 001 (2010). arXiv:1007.0482 [astro-ph.CO]

38. H. Motohashi, A.A. Starobinsky, J. Yokoyama, Future oscillations around phantom divide in $f(R)$ gravity. JCAP 06, 006 (2011). arXiv:1101.0744 [astro-ph.CO]

39. L.G. Jaime, L. Patio, M. Salgado, Note on the equation of state of geometric dark-energy in $f(R)$ gravity. Phys. Rev. D 89, 084010 (2014). arXiv:1312.5428 [gr-qc]

40. S.D. Odintsov, V.K. Oikonomou, Autonomous dynamical system approach for $f(R)$ gravity. Phys. Rev. D 96(10), 104049 (2017). arXiv: 1711.02230 [gr-qc]

41. S. Nojiri, S.D. Odintsov, Modified gravity with negative and positive powers of the curvature: Unification of the inflation and of the cosmic acceleration. Phys. Rev. D 68, 123512 (2003). arXiv:hep-th/0307288

42. S. Nojiri, S.D. Odintsov, Modified $f(R)$ gravity consistent with realistic cosmology: from matter dominated epoch to dark energy universe. Phys. Rev. D 74, 086005 (2006). arXiv:hep-th/0608008 